\title{
Self-Confidence and Paranoia: An Experimental Study Using an Immersive Virtual Reality Social Situation
}

\author{
Stephanie Atherton \\ University of Oxford, UK \\ Angus Antley \\ University of Oxford and Oxford Health NHS Foundation Trust, UK
}

Nicole Evans, Emma Cernis and Rachel Lister

University of Oxford, UK

Graham Dunn

University of Manchester, and MRC NW Hub for Trials Methodology Research, Manchester, UK

Mel Slater

University College London, UK and University of Barcelona, Spain

Daniel Freeman

University of Oxford, UK

\begin{abstract}
Background: Paranoia may build directly upon negative thoughts about the self. There have been few direct experimental tests of this hypothesis. Aims: The aim of the study was to test the immediate effects of manipulating self-esteem in individuals vulnerable to paranoia. Method: A two condition cross-over experimental test was conducted. The participants were 26 males reporting paranoid ideation in the past month. Each participant experienced a neutral immersive virtual reality (VR) social environment twice. Before VR participants received a low self-confidence manipulation or a high self-confidence manipulation. The order of
\end{abstract}

Reprint requests to Daniel Freeman, Oxford Cognitive Approaches to Psychosis, University Department of Psychiatry, University of Oxford, Warneford Hospital, Oxford OX3 7JX, UK. E-mail: daniel.freeman@psych.ox.ac.uk 
manipulation type was randomized. Paranoia about the VR avatars was assessed. Results: The low self-confidence manipulation, relative to the high self-confidence manipulation, led to significantly more negative social comparison in virtual reality and higher levels of paranoia. Conclusions: Level of self-confidence affects the occurrence of paranoia in vulnerable individuals. The clinical implication is that interventions designed to improve selfconfidence may reduce persecutory ideation.

Keywords: Delusions, persecutory delusions, self-esteem.

\section{Introduction}

Paranoid thoughts are increasingly recognized as a common phenomenon in the general population, with many people holding a few paranoid ideas and a few people holding many (Freeman et al., 2005; Bebbington et al., 2013; Wong, Freeman and Hughes, 2014). The implication for research is that studying individuals in the general population who experience paranoid thoughts can be informative for understanding clinical disorder. The key hypothesis studied in the current report is that paranoid thoughts build upon negative ideas about the self (Freeman et al., 2002). When an individual has negative self-esteem they are more likely to believe that they are inferior, apart, and different from others and therefore more vulnerable to harm. Paranoia may be an extension of such concerns.

Three recent systematic reviews indicate that negative beliefs about the self are associated with paranoia (Garety and Freeman, 2013; Kesting and Lincoln, 2013; Tiernan, Tracey and Shannon, 2014). In a study of 301 patients with psychosis over 12 months, negative beliefs about the self were a predictor of the persistence of persecutory delusions (Fowler et al., 2012). Similarly, a longitudinal study of 60 patients with persecutory delusions showed that negative beliefs about the self predicted the persistence of paranoia over 6 months (Vorontsova, Garety and Freeman, 2013). When self-esteem is improved in treatment then positive symptoms of psychosis decrease (e.g. Laithwaite et al., 2007).

There have, however, been only two experimental tests of the role of self-esteem in the occurrence of paranoid ideation. These have both indicated the potential causal role of negative self cognition. Lincoln, Hohenhaus and Hartmann (2013) tested an unselected group of 71 students. The participants were randomly assigned to a brief compassionfocused intervention targeting negative emotions and low self-esteem or a control condition. They were then encouraged to re-experience a distressing memory. Participants in the intervention group reported significantly higher self-esteem, which accounted for a reduction in the frequency of paranoid thoughts. Freeman, Evans et al. (2014) tested 60 females with paranoid thoughts in the past month (i.e. an analogue population). Paranoia was assessed by participants' interpretations of a neutral immersive virtual reality social situation. Self-concept was manipulated by altering the participants' height in VR, since height is a marker of social status. It was found that reducing a person's height caused more negative comparison of the self in relation to others that fully accounted for an increase in paranoia.

The current study followed on from that by Freeman, Evans et al. (2014). This time self-esteem was manipulated more directly before experiencing virtual reality. The effects of high self-confidence and low self-confidence manipulations on paranoia in virtual reality were tested. It was predicted that a low self-confidence manipulation, relative to a high selfconfidence manipulation, would result in more negative appraisals of the self in relation to others and greater levels of paranoia. 


\begin{abstract}
Method
Participants

Two hundred and forty-two potential participants from the general population were screened for current persecutory ideation. They were identified from the screening stage of a different experimental study (see Freeman, Dunn et al., 2014) in which they had not participated. To meet inclusion criteria, participants had to be: aged between 18 and 70; male; report paranoid thinking in the past month as assessed by a score of 17 or above on the Green et al. (2008) Paranoid Thoughts Scale Part B (GPTS-B). The 26 participants had a mean age of 43.4 years $(S D=16.3)$, and a mean GPTS-B score of $24.2(S D=13.1)$.
\end{abstract}

\title{
Design
}

The study had a within-subjects design, whereby all participants experienced both conditions of the independent variable of confidence induction (high and low self-confidence). The participants were allocated to whichever condition they would complete first using a simple randomization procedure. There was a 5-minute break between the two testing conditions. This study had received approval from the University of Oxford's research ethics committee.

\section{Self-confidence inductions}

The inductions were devised for this study. In the high self-confidence condition participants were told to think of a time in their life that they established as the most self-confident they had ever been. They were provided with a list of 16 "confident qualities" to aid them (e.g. Admired, Skilled, Intelligent). They were asked why they identified that time as their most self-confident, how that self-confidence felt, and what emotions they associated with it. The interviewer then asked the participant to choose five qualities from the list that represented their characteristics at that time (or to add extra words, if necessary). Participants explained why they had chosen each word in particular, giving if possible examples of having displayed that characteristic, before narrowing down to the three most defining qualities. They then took part in a visualization task with the interviewer, designed to aid induction of their past feelings of high self-confidence. Participants first visualized themselves at the time they had chosen, and elaborated on the associated emotions, with the help of the interviewer. Next, they were asked to imagine that they, at that most self-confident time, had been invited to a gathering, and were talking to a stranger. Participants were asked questions about how they felt in the imagined situation, what they perceived about the unknown person, and what they believed the person might be thinking about them. The low self-confidence condition used the same procedures but this time focused on a time that the participants established as the least self-confident they have ever been, and the word list presented comprised the opposites of the qualities noted above (e.g. Disrespected, Unskilled, Unintelligent). Visualization also concerned this negative self-image.

\section{Assessments}

Paranoid Thoughts Scale Part B (GPTS-B; Green et al., 2008). This scale, used for screening participants, measures persecutory thinking over the past month. It consists of 
16 statements concerning paranoid thoughts about others (e.g. "I have definitely been persecuted"; "I was distressed by people wanting to harm me in some way"). Statements are rated on a 5-point scale, where: $1=$ Not at all; $3=$ Somewhat; $5=$ Totally. Higher scores indicate greater levels of paranoia

Visual analogue scale (VAS) of confidence. At the beginning of the study, and following each confidence induction procedure, participants marked on a scale of 0 to $100(0=$ Not at all, $50=$ Moderately, $100=$ Totally) how confident they felt "right now".

Social Comparison Scale (SCS; Allan and Gilbert, 1995). This was used to assess how participants compared themselves to computer avatarsin VR. It contained 14 bipolar scale items (e.g. Inferior-Superior, Left out-Accepted), which included three extra items: Less powerful-More powerful; Less respected-More respected; Less able to harm-More able to harm. These were rated on a scale of 1 to 10, which represented the bipolar ends of the scale. Higher scores indicated the perception of a greater social rank than the avatars. In the current study, the internal consistency of this scale was high (Cronbach's $\alpha=.85$ ).

State Social Paranoia Scale (SSPS; Freeman et al., 2007). This scale was used to measure paranoid thoughts about the avatars in VR. It consists of 10 persecutory ideation items (e.g. "Someone was hostile towards me"; "Someone was trying to intimidate me"). Statements were rated on a 5-point scale, where: $1=$ Do not agree; $2=$ Agree a little; $3=$ Agree moderately; $4=$ Agree very much; $5=$ Totally agree. Higher scores indicated greater persecutory ideation. The internal consistency of this scale was high (Cronbach's $\alpha=.90$ ).

\section{Virtual reality}

The equipment used was identical to that in Freeman, Evans et al. (2014). Participants viewed the VR environment using an NVIS SX111 head mounted display (HMD), which has dual displays presenting images to each eye. The HMD has an $111^{\circ}$ diagonal binocular field of view, with a resolution of $1280 \times 1024$ in each eye and a refresh rate of the images presented to each eye of $60 \mathrm{~Hz}$. Audio of the underground was presented over in-built headphones. The HMD was driven from a Windows XP PC. The participant's head position and orientation was monitored by an Intersense IS900 tracker attached to the HMD, which fed back to ceiling sensors. This data were read by a Virtual Reality Peripheral Network (VRPN) IS900 server application. However, due to technical difficulties with this HMD, it was replaced partway through the study with the VR1280 HMD (as used in Freeman et al., 2008). With this, the field of view was reduced to $60^{\circ}$, but all other HMD specifications remained the same, as did the method of tracking. The change of HMD did not affect levels of paranoia in either induction condition $(p>.05)$.

The environment used was the same as that created for Freeman, Evans et al. (2014). The same VR scenario was used in both confidence induction conditions, consisting of a 6-minute London underground tube journey with three stops at platforms. The virtual platform and tube train cars were created using Maya, and rendered using the XVR application platform. The software for this platform was run on a Windows XP PC, which had a Nvidia GTX580 $3 \mathrm{D}$ graphics card to generate the images in the HMD dual displays. There were 21 other virtual characters, for which Rocket Box avatars were used. Their movements were modelled on real human movements recorded using Arena OptiTrack motion capture software, which 




Figure 1. (Colour online) View inside the virtual tube train

were edited in MotionBuilder and applied to the avatar models using the hardware accelerated library for character animation (HALCA) (Spanlang, 2009). This included breathing motions and a randomization of gaze direction. Several avatars were additionally programmed to look towards the participant when they were oriented towards them, but their expressions remained neutral across conditions. The audio used was pre-recorded background conversation, overlaid with the soundtrack of the tube journey (e.g. car doors opening and closing). An image of the VR situation is provided in Figure 1.

\section{Analysis}

Data from this two-period crossover trial were analyzed using Statal3 (StataCorp, 2013). There were no missing data. Although these data could be analyzed through a repeated measures analysis of variance, a simpler but mathematical equivalent procedure is to look at a simple linear regression model to evaluate the effect of order of the self-confidence inductions on the change in the outcome variable from period one to period two (or, more accurately, the change score divided by half so that the resulting regression coefficient is interpreted as the effect of the experimental condition - i.e. self-confidence induction - on outcome). The logic and procedure for doing this, including the demonstration that the difference between the two groups defined by their treatment order is an estimate of twice the treatment effect 
Table 1. Results: summary statistics: mean $(S D)$

\begin{tabular}{lllll}
\hline Outcome & Order $^{*}$ & Baseline & Time 1 & Time 2 \\
\hline Visual Analogue & High SCI first $(n=13)$ & $74.08(16.58)$ & $76.62(15.71)$ & $50.69(21.01)$ \\
Scale (VAS) & Low SCI first $(n=13)$ & $78.38(14.92)$ & $44.15(19.41)$ & $85.85(14.75)$ \\
Social Comparison & High SCI first $(n=13)$ & & $85.08(15.10)$ & $59.15(16.99)$ \\
Scale (SCS) & Low SCI first $(n=13)$ & & $52.38(10.69)$ & $95.54(8.65)$ \\
State Social Paranoia & High SCI first $(n=13)$ & & $11.38(2.10)$ & $14.69(7.98)$ \\
Scale (SSPS) & Low SCI first $(n=13)$ & & $14.54(4.50)$ & $11.85(5.79)$ \\
\hline
\end{tabular}

*SCI: Self-Confidence Induction

(the rationale for dividing the change by 2, above) is explained by Armitage, Berry and Matthews. (2002, page 631). Significance test results for all the analyses are quoted as twotailed probabilities. This procedure was first carried out on the VAS confidence ratings, in order to verify that the induction procedures had been effective, and then on the results from the Social Comparison Scale (SCS) and the State Social Paranoia Scale (SSPS).

\section{Results}

\section{The self-confidence inductions}

Summary statistics are provided in Table 1 . It can be seen from the Table that compared to the baseline, the low self-confidence condition lowered the VAS scores considerably but the effect of the high self-confidence condition was much less marked. However, it is the contrast between the high and low self-confidence conditions that is of relevance here. Examination of the means at time 1 and time 2 for the VAS data presented in Table 1 indicates a clear difference between these two conditions. The effect of the low induction condition compared to the high induction condition was highly statistically-significant and was estimated to be -33.81 (SE 4.91; 95\% CI -43.43 to $-24.18 ; p<.001$ ).

\section{Paranoia in VR}

Table 1 illustrates a consistent effect of the low self-confidence induction on the mean paranoia scores (SSPS) - low self-confidence being associated with greater paranoia. The effect of the low induction condition compared to the high was estimated to be +3.00 (SE 1.30; 95\% CI +0.45 to $+5.55 ; p=.021$ ). A list of spontaneous statements from participants with regards to paranoia is presented in Table 2 .

\section{Social comparison in $V R$}

The SCS scores were considerably lower in the low self-confidence condition than in the high self-confidence condition. The effect was highly statistically-significant and was estimated to be -34.53 (SE 2.88; 95\% CI -40.18 to -28.89 ; $p<.001$ ).

\section{Discussion}

This study used virtual reality to examine the interpretation of a neutral social situation by individuals vulnerable to paranoid ideation i.e. an analogue population. It tested the causal 
Table 2. Spontaneous participant comments concerning paranoid ideation

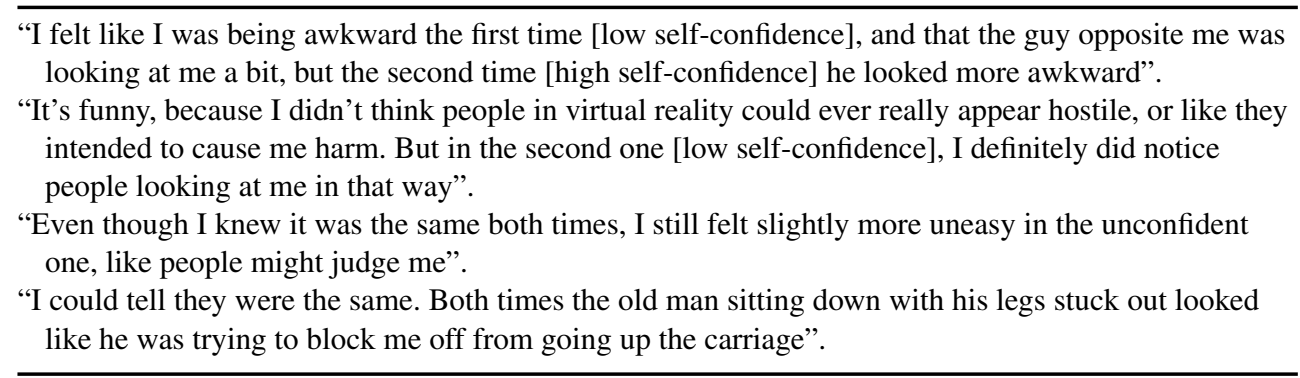

role of self-esteem, by manipulating whether the person felt self-confident or unconfident before entering virtual reality. The findings were very clear: going into the environment with low self-confidence, relative to high self-confidence, led to more negative views of the self in relation to others and greater levels of paranoia. The results support the hypothesis in a cognitive model that paranoia builds upon negative views of the self (Freeman et al., 2002; Freeman and Garety, 2014), and it is consistent with observational (e.g. Fowler et al., 2012) and experimental study results (e.g. Freeman, Evans et al., 2014; Lincoln et al., 2013). Tests of self-esteem interventions in patients with clinical paranoia are warranted (Freeman, Pugh et al., 2014).

There are several limitations to the study. Although the use of a social situation has ecological validity, the effects of self-esteem were only tested during a short period of time. The sample size was small, although the effects of the manipulations were moderate to large. In a two-period crossover trial such as this, valid statistical inferences are also dependent on the assumption of no carry-over effects. The study was only carried out with one gender, so the applicability to females was not established. Further, the addition of a neutral condition would have aided in the interpretation of the results. Unlike the study of Freeman, Evans et al. (2014) we did not test social comparison scores as mediators of paranoia scores in VR; this is because in the current study self-esteem was directly manipulated and therefore this additional analysis was unnecessary. Changes in social comparison merely provided a further check for the selfconfidence manipulations. Despite the limitations, the study clearly provides causal evidence for a direct role of negative views about the self in the occurrence of paranoid ideation.

\section{Acknowledgements}

The study was supported by a Medical Research Council (MRC) Senior Clinical Fellowship awarded to Daniel Freeman (G0902308). Mel Slater is supported by the ERC Advanced Grant TRAVERSE227985.

\section{References}

Allan, S. and Gilbert, P. (1995). A social comparison scale: psychometric properties and relationship to psychopathology. Personality and Individual Differences, 19, 293-299. doi: 10.1016/0191-8869(95)00086-L

Armitage, P., Berry, G. and Matthews, J. N. S. (2002). Statistical Methods in Medical Research (4 ${ }^{\text {th }}$ $e d n)$. Oxford: Blackwell. 
Bebbington, P., McBride, O., Steel, C., Kuipers, E., Radovanovic, M., Brugha, T., et al. (2013). The structure of paranoia in the general population. British Journal of Psychiatry, 202, 419427.

Fowler, D., Hodgekins, J., Garety, P., Freeman, D., Kuipers, E., Dunn, G., et al. (2012). Negative cognition, depressed mood and paranoia: a longitudinal pathway analysis using structural equation modelling. Schizophrenia Bulletin, 38, 1063-1073.

Freeman, D., Dunn, G., Murray, R., Evans, N., Lister, R., Antley, A., et al. (2014). How cannabis causes paranoia: using the intravenous administration of $\Delta^{9}$-tetrahydrocannabinol (THC) to identify key cognitive mechanisms leading to paranoia. Schizophrenia Bulletin. doi: 10.1093/schbul/ sbu098

Freeman, D., Evans, N., Lister, R., Antley, A., Dunn, G. and Slater, M. (2014). Height, social comparison, and paranoia: an immersive virtual reality experimental study. Psychiatry Research, 218, 348-352.

Freeman, D. and Garety, P. (2014). Advances in understanding and treating persecutory delusions. Social Psychiatry and Psychiatric Epidemiology, 49, 1179-1189.

Freeman, D., Garety, P. A., Bebbington, P. E., Smith, B., Rollinson, R., Fowler, D., et al. (2005). Psychological investigation of the structure of paranoia in a non-clinical population. British Journal of Psychiatry, 186, 427-435.

Freeman, D., Garety, P. A., Kuipers, E., Fowler, D. and Bebbington, P. E. (2002). A cognitive model of persecutory delusions. British Journal of Clinical Psychology, 41, 331-347. doi: 10.1348/014466502760387461

Freeman, D., Pugh, K., Antley, A., Slater, M., Bebbington, P., Gittins, M., et al. (2008). A virtual reality study of paranoid thinking in the general population. British Journal of Psychiatry, 192, 258263.

Freeman, D., Pugh, K., Dunn, G., Evans, N., Sheaves, B., Waite, F., et al. (2014). An early Phase II randomized controlled trial testing the effect on persecutory delusions of using CBT to reduce negative cognitions about the self: the potential benefits of enhancing self confidence. Manuscript submitted for publication.

Freeman, D., Pugh, K., Green, C., Valmaggia, L., Dunn, G. and Garety, P. (2007). A measure of state persecutory ideation for experimental studies. The Journal of Nervous and Mental Disease, 195, 781-784. doi: 10.1097/NMD.0b013e318145a0a9

Garety, P. A. and Freeman, D. (2013). The past and future of delusions research: from the inexplicable to the treatable. The British Journal of Psychiatry, 203, 327-333. doi: 10.1192/bjp.bp.113.126953

Green, C. E. L., Freeman, D., Kuipers, E., Bebbington, P., Fowler, D., Dunn, G., et al. (2008). Measuring ideas of persecution and social reference: the Green et al. Paranoid Thought Scales (GPTS). Psychological Medicine, 38, 101-111.

Kesting, M. and Lincoln, T. (2013). The relevance of self-esteem and self-schemas to persecutory delusions. Comprehensive Psychiatry, 54, 766-789.

Laithwaite, H. M., Gumley, A., Benn, A., Scott, E., Downey, K., Black, K., et al. (2007). Self-esteem and psychosis: a pilot study investigating the effectiveness of a self-esteem programme on the selfesteem and positive symptomatology of mentally disordered offenders. Behavioural and Cognitive Psychotherapy, 35, 569-577.

Lincoln, T. M., Hohenhaus, F. and Hartmann, M. (2013). Can paranoid thoughts be reduced by targeting negative emotions and self-esteem? An experimental investigation of a brief compassionfocused intervention. Cognitive Therapy and Research, 37, 390-402.

Spanlang, B. (2009). HALCA hardware accelerated library for character animation. Barcelona: Universitat de Barcelona, EVENT Lab.

StataCorp (2013). Stata Statistical Software: Release 13. College Station, TX: StataCorp LP.

Tiernan, B., Tracey, R. and Shannon, C. (2014). Paranoia and self-concepts in psychosis. Psychiatry Research. doi: 10.1016/j.psychres.2014.02.003 
Vorontsova, N., Garety, P. and Freeman, D. (2013). Cognitive factors maintaining persecutory delusions in psychosis: the contribution of depression. Journal of Abnormal Psychology, 122, 11211131.

Wong, K., Freeman, D. and Hughes, C. (2014). Suspicious young minds: a study of two and a half thousand 8-14 year olds in the UK and Hong Kong. British Journal of Psychiatry, 205, 221-229. 